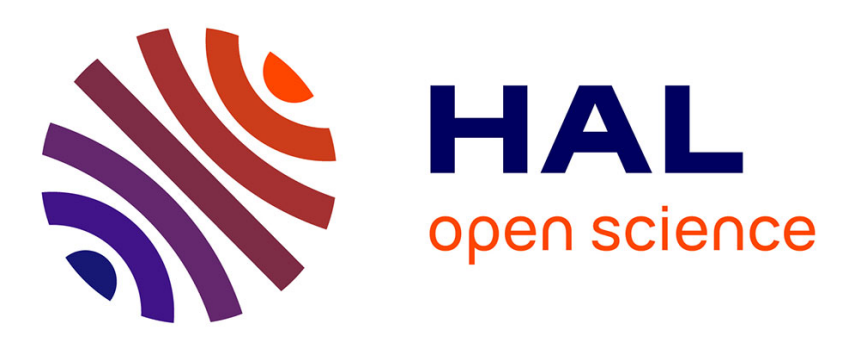

\title{
Influence of chemical surface modification of cellulose nanowhiskers on thermal, mechanical, and barrier properties of poly(lactide) based
}

Etzael Espino-Pérez, Julien Bras, Violette J. Ducruet, Alain Guinault, Alain Dufresne, Sandra Domenek

\section{To cite this version:}

Etzael Espino-Pérez, Julien Bras, Violette J. Ducruet, Alain Guinault, Alain Dufresne, et al.. Influence of chemical surface modification of cellulose nanowhiskers on thermal, mechanical, and barrier properties of poly(lactide) based. European Polymer Journal, 2013, 49 (10), pp.3144-3154. 10.1016/j.eurpolymj.2013.07.017 . hal-02350665

\section{HAL Id: hal-02350665 https://hal.science/hal-02350665}

Submitted on 10 Jan 2020

HAL is a multi-disciplinary open access archive for the deposit and dissemination of scientific research documents, whether they are published or not. The documents may come from teaching and research institutions in France or abroad, or from public or private research centers.
L'archive ouverte pluridisciplinaire HAL, est destinée au dépôt et à la diffusion de documents scientifiques de niveau recherche, publiés ou non, émanant des établissements d'enseignement et de recherche français ou étrangers, des laboratoires publics ou privés. 
Macromolecular Nanotechnology

\title{
Influence of chemical surface modification of cellulose nanowhiskers on thermal, mechanical, and barrier properties of poly(lactide) based bionanocomposites
}

\author{
Etzael Espino-Pérez ${ }^{\mathrm{a}, \mathrm{b}}$, Julien Bras ${ }^{\mathrm{a}}$, Violette Ducruet ${ }^{\mathrm{c}}$, Alain Guinault ${ }^{\mathrm{d}}$, Alain Dufresne ${ }^{\mathrm{a}}$, \\ Sandra Domenek ${ }^{\mathrm{b}, *}$ \\ a LGP2/Grenoble INP-Pagora/CNRS, 461 rue de la papeterie, Domaine universitaire, C10065, 38402 Saint Martin d'Hères Cedex, France

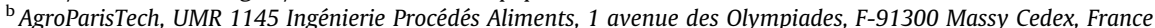 \\ ' INRA, UMR 1145 Ingénierie Procédés Aliments, 1 avenue des Olympiades, F-91300 Massy Cedex, France \\ d CNAM, PIMM, Laboratoire Procédés Ingénierie en Mécanique et Matériaux, 151 boulevard de l'hôpital, F-75013 Paris, France
}

Keywords:

Cellulose nanocrystals

Poly(lactic acid)

Mechanical properties

Crystallization

Barrier properties

Surface modification

\begin{abstract}
A B S T R A C T
In the aim of producing fully organic bionanocomposite based on poly(lactide) (PLA), cellulose nanowhiskers (CNW) were grafted by $n$-octadecyl-isocyanate (CNW-ICN) applying an in situ surface grafting method. The compatibilizing effect of the long aliphatic grafted chain was investigated by thermal, mechanical and permeability analysis of solvent cast nanocomposite films. The grafted CNW-ICN could be successfully dispersed in the polymer matrix. The gained compatibility brought about a nucleating effect, decreasing the half time of isothermal crystallization from $25 \mathrm{~min}$ for the neat PLA to $8.4 \mathrm{~min}$ for the nanocomposite including $2.5 \mathrm{wt} \% \mathrm{CNW}$-ICN, e.g., tensile strength was improved by $10 \mathrm{MPa}$ for the same $2.5 \mathrm{wt} \% \mathrm{CNW}$-ICN/PLA composite. Mechanical reinforcement was also effective in the rubbery state of PLA and increased the tensile modulus of the rubbery plateau providing thereby thermal resistance to the polymer. Oxygen barrier properties did not change significantly upon the inclusion of CNW-ICN, even when the quantity of CNW-ICN was increased to $15 \mathrm{wt} \%$. More interestingly, the water vapour permeability of the CNW-ICN nanocomposite was always lower than the one of ungrafted CNW composites, which led to the conclusion that the hydrophobic surface graft and improved compatibility could counteract the effect of inclusion of hydrophilic structures in the matrix on water vapour transport. In conclusion, the surface grafting of CNW with isocyanates might be an easy and versatile tool for designing fully organic bionanocomposites with tailored properties. (c)
\end{abstract}

\section{Introduction}

Nowadays, driven by a growing consciousness for the environment and limited fossil carbon sources, there is a great interest in developing effective "green materials" derived from renewable sources being biodegradable and non-toxic to humans and environment. To enhance the

\footnotetext{
* Corresponding author. Address: AgroParisTech, UMR 1145, 1 rue des Olympiades, F-91744 Massy Cedex, France. Tel.: +33 169935068.

E-mail address: sandra.domenek@agroparistech.fr (S. Domenek).
}

performance of those materials in technological terms, in particular thermal, mechanical and barrier properties, the compounding with other polymers or the use of filler are common strategies. Cellulose nanowhiskers (CNW) are biobased and very stiff filler [1], that's why their use in nanocomposites has been firstly tested in the 1990s [2]. Since that time, they have been used increasingly as nanofiller in a number of different applications and in a number of polymers, which has already been extensively reviewed [3]. To give some examples of large literature, CNW has been used in natural rubber [4,5], starch [6], cellulose 
acetate [7], poly(ethylene oxide) [8], poly(vinyl alcohol) [9], poly(caprolactone) (PCL) [10,11], poly(3-hydroxybutyrate-hydroxyvalerate) (PHBV) [12,13] or poly(lactide) [11,14-17]. Among those polymers, poly(lactide) (PLA), which is a biobased and biodegradable polymer, has attracted large research interest due to its promising properties, its ease of processing and its industrial availability, as acknowledged by the increasing number of reviews and book chapters [18-21]. The dispersion of CNW or microfibrillated cellulose in PLA has already been studied and brings about several advantages, such as increased biodegradability [22], mechanical reinforcement [6,23-28] and modulated barrier properties [11,15]. One of the main drawbacks of $\mathrm{CNW}$ is their high propensity to agglomeration (or film forming) as soon as they are dried, even if a new drying-redispersion method by cation exchange of the $\mathrm{H}^{+}$counterions of $\mathrm{CNW}$ to $\mathrm{Na}^{+}$counterions has been very recently proposed [29]. Furthermore, CNW show poor compatibility with hydrophobic polymer matrices. Both properties stem from the difference in polarity caused by the hydrophilic character of the nanocrystal surface.

Different strategies are available to remedy this problem, such as the use of surfactants, encapsulation or grafting of the cellulose nanowhiskers surface. Several grafting strategies have already been used, for example surface acetylation of microfibrillar cellulose [30,31], reactive compatibilisation [32], or grafting of PLA oligomers onto the CNW surface [16,33]. Recently the grafting of long aliphatic chains onto CNW with isocyanate to modify the nanofiller/PCL matrix interaction has been proposed along with an innovative method of in situ surface grafting, and the beneficial effect on mechanical properties of the nanocomposite due to augmented compatibility has been clearly established [34]. The positive impact of the chemically modified CNW on nucleation of PCL and its crystallinity has also been studied in detail [10]. The isocyanate grafting reaction is having a number of advantages, such as high reaction rates, the absence of secondary products and the chemical stability of the urethane linkage [35].

The compatibilizing of PLA with CNW by surface grafting with long aliphatic isocyanate chain appears thus to be a versatile method for obtaining tailored bionanocomposites. The aim of this study was to apply this strategy to produce compatibilized PLA/CNW bionanocomposites. For that the CNW surface was modified by in situ surface grafting and characterized before producing PLA/CNW samples by solvent casting. Thermal, mechanical and barrier properties of ensuing material have then been evaluated in detail for the first time with a focus on the interaction between crystallinity, reinforcement and barrier properties.

\section{Experimental section}

\subsection{Materials}

Poly(lactide) (PLA) with a D content less than 5\%, INGEO 2002D, was purchased from NatureWorks (USA). The PLA pellets were dried under vacuum and stored over $\mathrm{P}_{2} \mathrm{O}_{5}$ at room temperature before use. Microcrystalline cellulose (MCC) powder was acquired from Sigma-Aldrich (France) and was used as a raw material for the production of cellulose nanowhiskers. Sulphuric acid (95\%) and n-octadecyl isocyanate were obtained from Sigma-Aldrich (France). Ethanol, acetone, chloroform, toluene, and dichloromethane were purchased from Chimie-Plus (France) and used as delivered.

\subsubsection{Cellulose nanowhiskers production}

The preparation of CNW followed an adaptation of the optimized protocol of Bondeson et al. [36]. In brief, $270.3 \mathrm{~g}$ of water were added to $56.9 \mathrm{~g}$ of MCC and mixed until good dispersion. Thereafter $529.7 \mathrm{~g}$ of sulphuric acid was slowly added to the suspension (65 wt\% acid concentration). The MCC in the acid suspension was then hydrolysed at $44{ }^{\circ} \mathrm{C}$ for $130 \mathrm{~min}$ under mechanical stirring. The excess of sulphuric acid was removed by repeated cycles of water exchange/centrifugations $(10,000 \mathrm{rpm})$ at $4{ }^{\circ} \mathrm{C}$ for $10 \mathrm{~min}$, until the supernatant became turbid. After the centrifugation cycles, the suspension containing the CNW was dialyzed in distilled water during one week. Then the CNW suspension was homogenized using an Ultra-Turax T25 homogenizer (France) and was subjected to ultrasound treatment in order to ensure dispersion of CNW. Finally, the neutralization was carried out by adding drops of a $1 \mathrm{wt} \% \mathrm{NaOH}$ solution to the $\mathrm{CNW}$ suspension. Some drops of chloroform were added to avoid microbiological development. Suspensions were stored at $4{ }^{\circ} \mathrm{C}$.

\subsubsection{Chemical modification of cellulose nanowhiskers}

CNW were modified with $n$-octadecyl isocyanate (ICN) according to a protocol adapted from Siqueira et al. [34]. The CNW dispersed in dry toluene were grafted with ICN, 10 equivalents according to the hydroxyl groups available at the surface of the cellulose nanoparticles ( $10 \%)$, under nitrogen atmosphere at $110^{\circ} \mathrm{C}$ using dibutyl dilauryl as catalyst. The reaction system was washed by centrifugation/redispersion for amines and unreacted isocyanates removal. Finally the modified CNW (named hereafter CNWICN) was stored in chloroform.

\subsubsection{Processing of bionanocomposite films}

PLA/CNW and PLA/CNW-ICN nanocomposite films were prepared by the solvent casting method. For that, the CNW and CNW-ICN suspensions were solvent exchanged to chloroform by centrifugation. Chloroform suspensions at concentrations of $5 \mathrm{wt} \%, 14 \mathrm{wt} \%$ and $30 \mathrm{wt} \%$ of CNW and CNW-ICN were prepared and sonicated during $10 \mathrm{~min}$. Thereafter, these suspensions were mixed with a PLA/chloroform solution ( $7 \mathrm{wt} \%$ ) to get a final concentration of cellulose nanowhiskers of $2.5 \mathrm{wt} \%, 7.5 \mathrm{wt} \%$ and $15 \mathrm{wt} \%$ in the PLA bionanocomposites. To ensure dispersion of the nanowhiskers in the PLA solution, the suspensions were mixed for $5 \mathrm{~h}$ at $60^{\circ} \mathrm{C}$ and sonicated twice during $10 \mathrm{~min}$. Subsequently the suspensions were cast into Teflon dishes, left to evaporate at room temperature for one day, and then dried under vacuum until constant weight. Film thickness varied between 40 and $120 \mu \mathrm{m}$ according to the quantity cast into the Teflon dish. 


\subsection{Characterization methods}

The grafting of CNW was checked with the help of Attenuated Total Reflectance-Fourier Transform Infrared Spectroscopy (ATR-FTIR) (PerkinElmer, Spectrum 65). Spectra were recorded by depositing the dried film or powder of the CNW or CNW-ICN directly on the surface of the crystal. At least two measurements with different samples of each composition were performed to check reproducibility.

$\mathrm{X}$-ray analysis of CNW and CNW-ICN was performed using a Panalytical X'Pert Pro MPD-Ray diffractometer with Ni-filtered $\mathrm{Cu} K \alpha$ radiation $(\lambda=1.54 \AA$ ) generated at a voltage of $45 \mathrm{kV}$ and current of $40 \mathrm{~mA}$, and scan from $5^{\circ}$ to $60^{\circ}$. The crystallinity index was evaluated using the BuschleDiller and Zeronian Eq. (1):

$I_{c}=1-\frac{I_{1}}{I_{2}}$

where $I_{1}$ is the intensity at the minimum $\left(2 \theta=18.8^{\circ}\right)$ and $I_{2}$ is the intensity associated with the crystalline region of cellulose $\left(2 \theta=22.75^{\circ}\right)$. All analyses were duplicated.

Dynamic contact angle measurements on CNW and CNW-ICN were carried out with distillate water. A DataPhysics instrument (Anton Paar) equipped with a CCD camera was used, allowing the determination of contact angle at equilibrium with a precision of $\pm 1^{\circ}$. The kinetic of its evolution started with a few tens of milliseconds after the deposition of the drop by taking up to 1000 images per second. Each measurement was the average value of at least triplicate determination.

CNW and CNW-ICN were further characterized by field emission scanning electron microscopy (FE-SEM), model Quanta $200 \mathrm{FEI}$, with an accelerating voltage of $12.5 \mathrm{kV}$. Samples were put onto a substrate with carbon tape and coated with a thin gold layer. Digital image analysis (ImageJ Software) was used in order to measure the dimensions of the nanoparticles, an average of at least 50 measures was proposed. SEM observation of film samples was realized after cryofracture in liquid nitrogen by means of a Zeiss EVO MA10 and with an accelerating voltage of $7.05 \mathrm{kV}$.

Determination of the degree of crystallinity of PLA was performed on a DSC Q100 (TA Instrument) under nitrogen atmosphere calibrated with zinc and indium standards. The samples (4-6 mg) were put into aluminium pans (TZero) and analysed by a heat-cool-heat scan at a scanning speed of $10^{\circ} \mathrm{C} \mathrm{min}{ }^{-1}$ from $-10^{\circ} \mathrm{C}$ to $190^{\circ} \mathrm{C}$. All experiments were carried out in duplicate. The degree of crystallinity was calculated from the enthalpy of the first heating scan from the following equation:

$\chi_{c}=\frac{\Delta H_{m}-\Delta H_{c c}}{\Delta H_{m}^{0}}$

where $\Delta H_{m}$ is the measured endothermic enthalpy of melting corrected by the concentration of CNW, $\Delta H_{c c}$ is the exothermic enthalpy of cold crystallization during the DSC heating scan and $\Delta H_{m}^{0}$ is the enthalpy of fusion of repeating unit of the perfect crystal of infinite size of PLA, being $93 \mathrm{~J} \mathrm{~g}^{-1}$ [37]. The glass transition temperature of the bionanocomposites was determined during the second heating scan, after erasing of the thermal history.
Isothermal crystallization experiments at temperatures between 90 and $120^{\circ} \mathrm{C}$ were carried out on a DSC 1 (Mettler Toledo, France), calibrated with indium and zinc standards. Samples were melted at $190^{\circ} \mathrm{C}$ for $5 \mathrm{~min}$ and then quenched at the maximum cooling rate (approximately $50{ }^{\circ} \mathrm{C} \mathrm{min}-1$ ) to the desired crystallization temperature. Samples were maintained at crystallization temperature until the baseline was recovered (typically $3 \mathrm{~h}$ ). Subsequently, samples were quenched below glass transition to $-10^{\circ} \mathrm{C}$ and reheated with a scanning rate of $10^{\circ} \mathrm{C} \mathrm{min}^{-1}$ to $190^{\circ} \mathrm{C}$ to record glass transition and the melting peak. Measurements were done in duplicate. For the determination of the crystallization half time, the crystallization peak was integrated. The half time was taken at the time the integral reached $50 \%$ of its total value.

Measurement of the thermomechanical properties was carried out with a DMA RSA3 (TA Instruments, USA) equipment working in tensile mode. The measurements were performed at a constant frequency of $1 \mathrm{~Hz}$ and strain amplitude of $0.05 \%$ with a distance between jaws of $10 \mathrm{~mm}$. Film thickness was determined before each measurement. Firstly, the samples were heated from room temperature $\left(\sim 28^{\circ} \mathrm{C}\right)$ to $110^{\circ} \mathrm{C}$ and maintained at this temperature during $20 \mathrm{~min}$ in order to remove residual solvent eventually still present in the sample and to obtain a reproducible degree of crystallinity. Then the system was cooled down to $0{ }^{\circ} \mathrm{C}$ and the viscoelastic moduli during the heating run were recorded up to $180^{\circ} \mathrm{C}$ at $2{ }^{\circ} \mathrm{C} \mathrm{min}^{-1}$. The temperature of the $\alpha$-relaxation which can be associated to the glass transition temperature was taken at the maximum of the peak of the damping factor $(\tan \delta)$. Two samples were used to characterize each film.

The tensile testing of film samples was carried out at room temperature and relative humidity at a cross head speed of $5 \mathrm{~mm} \mathrm{~min}^{-1}$ with an Instron tensile testing machine (Instron Model 4507) equipped with pneumatic jaws on type I BA dumbbell shaped samples. The thickness of the samples was determined before the experiment and varied from 90 to $120 \mu \mathrm{m}$. Each value is an average of 7 measurements.

The oxygen transmission rate (OTR) was monitored at $23{ }^{\circ} \mathrm{C}$ and $0 \%$ relative humidity with the help of a Systech 8001 (France) apparatus. Oxygen permeability was obtained by dividing respectively OTR by the film thickness. Experiments were carried out in duplicate.

The water vapour transmission rate (WVTR) of the films was measured according to the norm NF H 00-030 at $25{ }^{\circ} \mathrm{C}$ and $50 \% \mathrm{RH}$. The procedure consisted in measuring the weight uptake (balance Kern 770, precision $0.01 \mathrm{mg}$ ) of precedingly dried $\mathrm{CaCl}_{2}$ in a metal cap sealed by the sample films and tightened with the help of beeswax. The specific exchange surface was $50.24 \mathrm{~cm}^{2}$. For controlling the environmental conditions, the cups were placed in a climatic chamber (Vötsch industrietechnik, VCN100). The WVTR ( $\mathrm{g} \mathrm{m} \mathrm{m}^{-2} \mathrm{~s}^{-1}$ ) value can be obtained from the slope of the weight uptake through the following equation:

WVTR $=\frac{\text { slope } \cdot l}{S}$,

where slope corresponds to the slope of the plot of cumulated weight against time, $S$ to the exchange surface, and 
$l$ to the film thickness, determined before measurement. Given values were averages of two or three experiments. The WVTR was normalized to permeability $(P)$ to take into account the pressure gradient between each side of the film with the help of

$P_{\mathrm{H}_{2} \mathrm{O}}=\frac{W V T R}{\Delta p}$,

where $\Delta p$ is the gradient in partial pressure of water (Pa).

The statistical analysis was realized with a one-way Analysis of Variance (ANOVA). When the differences were significant $(p<0.05)$, Duncan's test was used to check the differences between pairs of groups and was carried out using XLSTAT-Pro 7.0 software (Addinsoft, Paris, France).

\section{Results and discussion}

\subsection{Morphological analysis of cellulose nanowhiskers}

CNW were obtained by acid hydrolysis of the amorphous phase of microcrystalline cellulose. After the hydrolysis procedure, the CNW suspension showed flow birefringence when observed undercrosss polarized light as presented in Fig. 1a. This indicates a chiral nematic liquid crystalline alignment and reveals the existence of nanostructures [1]. The morphology of CNW was confirmed by field emission-scanning electron microscopy (FE-SEM) (Fig. 1b). The average geometric characteristics of CNW were evaluated at $243.9 \pm 48.5 \mathrm{~nm}$ in length and $9.4 \pm 2.5 \mathrm{~nm}$ in diameter. It is in accordance with published nanocrystal dimensions [38]. After chemical grafting, structure was kept and similar dimension was achieved (Fig. 1c).

One of the drawbacks of CNW is their poor dispersibility in organic (hydrophobic) solvents and their propensity to aggregation because of the large number of hydroxyl groups on the surface [2]. In order to overcome this drawback, chemical modification of the CNW with an aliphatic grafting agent $n$-octadecyl isocyanate was carried out to provide hydrophobic behaviour to CNW. Fig. 2 shows the dispersion of CNW (Fig. 2a, tube a) and CNW-ICN (Fig. 2a, tube b) at $3 \mathrm{wt} \%$ in chloroform, being the solvent used for PLA dissolution. An improvement was clearly obtained after the CNW grafting since a homogenous suspension was observed.
ATR-FTIR analysis was carried out to confirm grafting of CNW. Fig. 2b shows that before the chemical treatment the CNW display characteristic bands of cellulose at $3320 \mathrm{~cm}^{-1}$ $(\mathrm{O}-\mathrm{H}), 1099 \mathrm{~cm}^{-1}$ (C-O of secondary alcohol), and 2919 and $2849 \mathrm{~cm}^{-1}$ ( $\mathrm{C}-\mathrm{H}$ from $\left.-\mathrm{CH}_{2}\right)$. After reaction, a strong increase of the band characteristic of the grafted alkyl chain $\left(-\mathrm{CH}_{3}\right.$ and $-\mathrm{CH}_{2}-$ groups) at 2919 and $2849 \mathrm{~cm}^{-1}$ was observed. The signal at $1697 \mathrm{~cm}^{-1}$ was interpreted as the presence of the ureic linkage formed during the reaction. The washing steps were effective, as the signal at $2260 \mathrm{~cm}^{-1}$ corresponding to the isocyanate functions was absent. Chemical grafting was thus clearly evidenced by the FTIR results.

The crystallinity index of both, CNW and CNW-ICN, was measured by X-ray scattering. X-ray diffraction measurements showed peaks located around $15.2^{\circ}$ and $22.8^{\circ}$ for $\mathrm{CNW}$ and two peaks around $15.3^{\circ}$ and $22.6^{\circ}$ for CNW-ICN. These peaks are characteristics of cellulose I. The crystallinity index was determined according to Eq. (1) showing values of $87.2 \%$ and $73.7 \%$ for CNW and CNW-ICN, respectively. The decrease may be explained by the fact that the surface grafting of the nanoparticle could induce a possible peeling effect of the modified CNW, as already observed for similar grafting [39]. Although the decrease observed, one can conclude that the crystalline structure of the nanoparticles was kept undamaged in the core after grafting with the $n$-octadecyl isocyanate.

Finally, the dynamic behaviour of the contact angle for a drop of distillate water on the surface of the cellulose particles was analysed to provide supplementary evidence on surface grafting. The CNW contact angle value was measured at $66^{\circ}\left( \pm 0.8^{\circ}\right)$. CNW-ICN displayed a significantly higher contact angle value at $89^{\circ}\left( \pm 0.7^{\circ}\right)$. This value also showed that the grafting density of cellulose nanowhiskers was probably low and there were still free hydroxyl groups on the surface. It was nevertheless enough to modify surface properties.

\subsection{Morphological analysis of bionanocomposites}

Fig. 3 shows the photographs of the different bionanocomposite films obtained by the casting process. Visual examination of the films indicated that good filler dispersion was achieved for the lowest CNW and CNW-ICN content $(2.5 \mathrm{wt} \%)$. The transparency of these two films can be considered like that of the neat biopolymer. The film
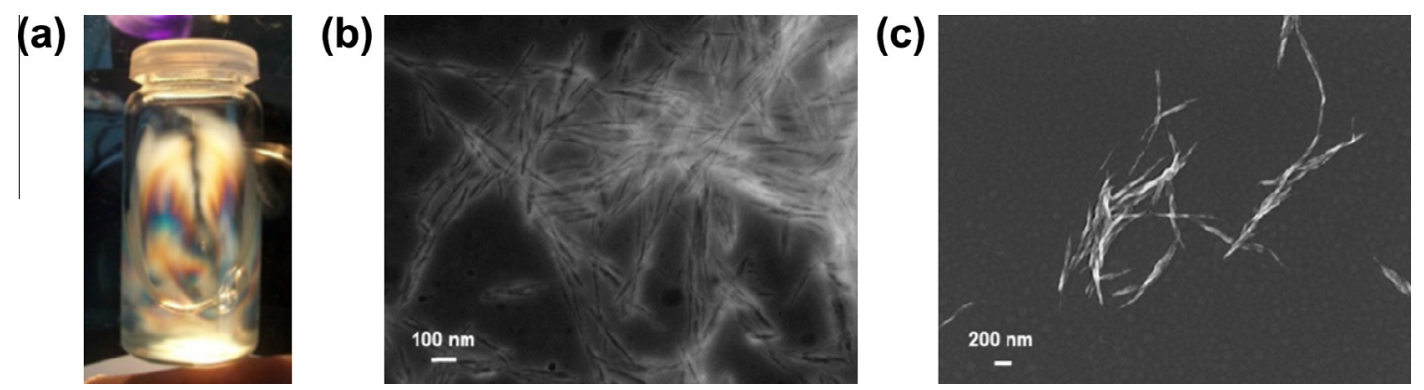

Fig. 1. (a) Birefringence behaviour of cellulose nanowhiskers, field emission scanning electron microscopy of (b) unmodified and (c) modified cellulose nanowhiskers. 

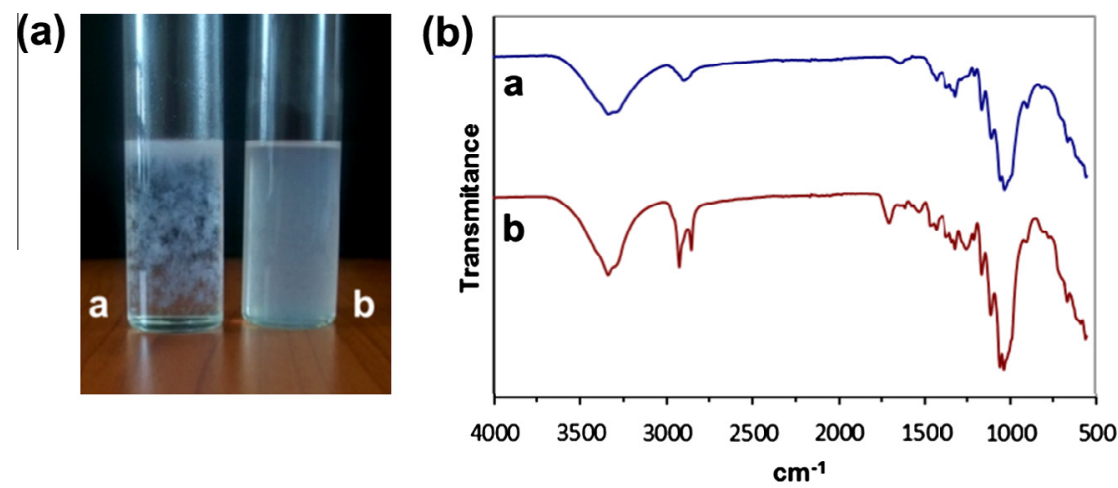

Fig. 2. (a) Dispersion of (a) unmodified and (b) modified cellulose nanowhiskers in chloroform, (b) FTIR spectrum for (a) unmodified and (b) modified cellulose nanowhiskers.

reinforced with $\mathrm{CNW}$-ICN was always more transparent (sample e in Fig. 3b) than the CNW containing film (sample b in Fig. 3a). At higher CNW loads, aggregation of the unmodified nanoparticles in the matrix can be observed giving rise to a granular structure (sample d in Fig. 3a). In the case of CNW-ICN, films became opaque but no aggregates were observed (sample $g$ in Fig. 3b). The difficulty to disperse unmodified CNW in PLA has already been evidenced in literature. For example Petersson and Oksman [40] showed loss in transparency for PLA/microcrystalline cellulose composites. Sanchez-Garcia et al. [11] had the same observation for PLA/cellulose fibre composites. More recently Fortunati et al. [41] showed the reduction of the transparency of PLA films due to dispersion of CNW with the help of a surfactant. For nearer examination of the film morphology, Fig. 4 shows cryofractured samples under SEM observation. A flat and neat fracture of neat PLA is seen. This result is comparable to literature data $[42,43]$. Upon dispersion of CNW, fracture surfaces became rough. At $2.5 \mathrm{wt} \%$ reinforcement in PLA a uniform distribution is obtained for both CNW (Fig. 4b) and CNW-ICN (Fig. 4e). For $15 \mathrm{wt} \%$ of nanofiller, aggregates of $\mathrm{CNW}$ and CNWICN were evidenced. However, the filler/matrix interaction was much better for CNW-ICN. Fig. 4d clearly proved that the interfacial adhesion between CNW aggregates and matrix was poor, and the aggregate insertion caused the formation of fissures around the filler. CNW-ICN appeared to be better distributed in PLA compared to CNW and adhesion to the PLA matrix seemed to be improved.

\subsection{Impact of CNW and CNW-ICN on crystallization properties of PLA}

The nanocomposites were analysed by differential scanning calorimetry with a heat-cool-heat cycle in order to investigate materials as produced and intrinsic properties after erasing of the thermal history of the samples. Measured thermal properties are summarized in Table 1 . The degree of crystallinity of the as produced samples calculated from the first heating scan of the cast samples showed that PLA and CNW-ICN samples had notably lower degrees of crystallinity than samples containing CNW. However, data were difficult to interpret because crystallization took place during the drying process of the casting solution, the kinetics of which is difficult to control. Therefore a more detailed investigation was carried out after erasing of the thermal history. The data obtained from the second heating run were, not surprisingly, much more reproducible. Typical thermograms of the second heating loop are shown in Fig. 5. The glass transition temperature of the bionanocomposites did not change significantly compared with that of the neat PLA (Table 1). On the contrary, Fortunati et al. [44] showed a decrease in the glass transition temperature of PLA including cellulose
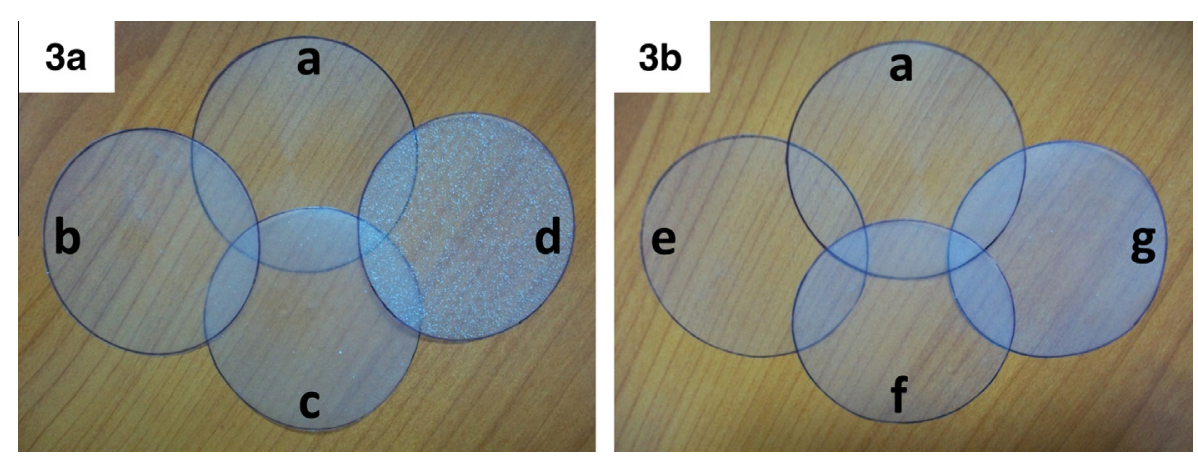

Fig. 3. Images of (a) neat PLA film compare with those of bionanocomposites reinforced in 3 (a) with CNW at (b) 2.5 wt\%, (c) 7.5 wt $\%$ and (d) 15 wt\%, and in 3(b) with CNW-ICN at (e) $2.5 \mathrm{wt} \%$, (f) $7.5 \mathrm{wt} \%$ and (g) $15 \mathrm{wt} \%$. 

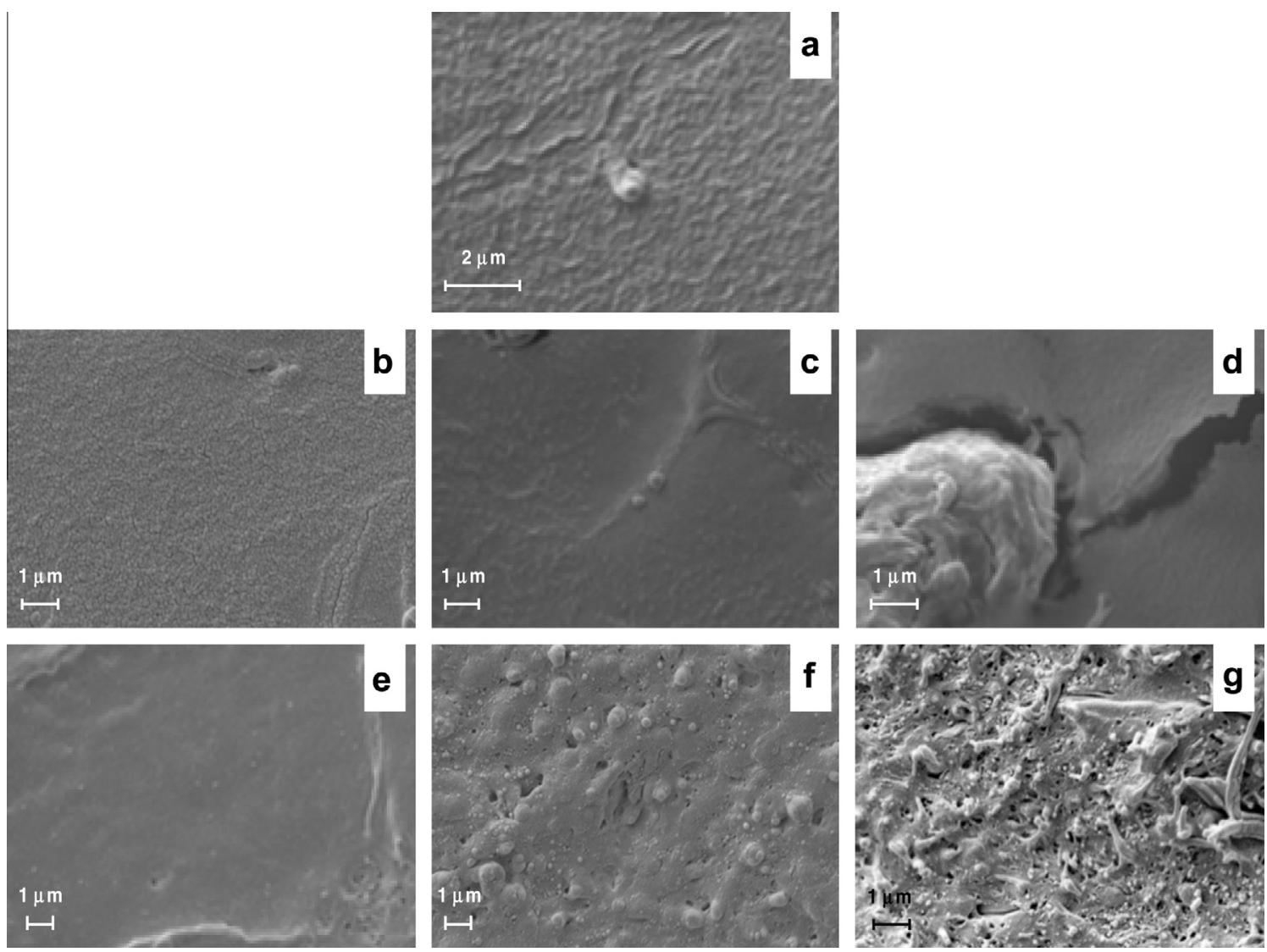

Fig. 4. Scanning electron microscopy (SEM) of (a) neat PLA and bionanocomposite cryofractured surfaces reinforced with CNW at (b) 2.5 wt\%, (c) 7.5 wt\% and (d) $15 \mathrm{wt} \%$, and CNW-ICN at (e) $2.5 \mathrm{wt} \%$, (f) $7.5 \mathrm{wt} \%$ and (g) $15 \mathrm{wt} \%$.

Table 1

Thermal properties of PLA and PLA bionanocomposites obtained from DSC at the first and second heating scan.

\begin{tabular}{llllllll}
\hline Sample & $\chi^{1}(\%)$ & $T_{\mathrm{g}^{2}}\left({ }^{\circ} \mathrm{C}\right)$ & $T_{\mathrm{cc}^{2}}\left({ }^{\circ} \mathrm{C}\right)$ & $\Delta H_{\mathrm{cc}^{2}}\left(\mathrm{~J} \mathrm{~g}^{-1}\right)$ & $T_{\mathrm{m}^{2}}\left({ }^{\circ} \mathrm{C}\right)$ & $\Delta H_{\mathrm{m}^{2}}\left(\mathrm{~J} \mathrm{~g}^{-1}\right)$ & $\Delta H_{\mathrm{m} \text { annealing }}{ }^{*}\left(\mathrm{~J} \mathrm{~g}^{-1}\right)$ \\
\hline PLA & 2.9 & 61 & - & - & 152.6 & - & 50 \\
PLA/CNW 2.5 wt\% & 12.4 & 59 & 127.7 & 10 & 152.2 & 10 & 42 \\
PLA/CNW 7.5 wt\% & 10.5 & 60 & - & - & 152.7 & - & 52 \\
PLA/CNW 15 wt\% & 17.2 & 60 & -2 & - & 152.2 & - & 41 \\
PLA/CNW-ICN 2.5 wt\% & 2.5 & 60 & 113.1 & 27 & 149.1 & 25 & 53 \\
PLA/CNW-ICN 7.5 wt\% & 6.6 & 60 & 113.8 & 24 & 150.4 & 21 & 52 \\
PLA/CNW-ICN 15 wt\% & 6.3 & 59 & 117.3 & 22 & 150.3 & 22 & 53 \\
\hline
\end{tabular}

Note: Enthalpy values correspond to PLA and are corrected by the content of CNW or CNW-ICN.

${ }^{1}$ Measuerd at the first heating scan.

${ }^{2}$ Measured at the second heating scan.

* After annealing for $3 \mathrm{~h}$ at $110^{\circ} \mathrm{C}$ from the melt at maximum crystallization rate.

nanocrystals and a surfactant for dispersion. A likely explication would be plasticizing of PLA by desorbed surfactants. Goffin et al. [16] showed also a decrease of $T_{\mathrm{g}}$ upon blending PLA with cellulose nanowhiskers modified by lactide oligomers. They conclude on plasticizing of the oligomers protruding in the PLA phase.

Furthermore, the PLA bionanocomposites reinforced with CNW-ICN showed a high cold crystallization peak, the peak temperature of which was reduced by approximately $10^{\circ} \mathrm{C}$ compared to the small peak of PLA containing $2.5 \mathrm{wt} \% \mathrm{CNW}$ (Fig. 5). The appearance of the high PLA cold crystallization peak showed that CNW, and more efficiently, the CNW-ICN were most likely acting as nucleating agents.

Therefore, the half time of isotherm crystallization from the melt, $t_{1 / 2}$, of the neat PLA and bionanocomposites was evaluated at different temperatures. Results are plotted out in Fig. 6. Half-time curves typically show a minimum around $110^{\circ} \mathrm{C}$. This temperature is known to be in the range of the optimum crystallization temperature of PLA, laying between 105 and $115^{\circ} \mathrm{C}$ [45-51]. Values of the half time of the pristine polymer are in agreement with 


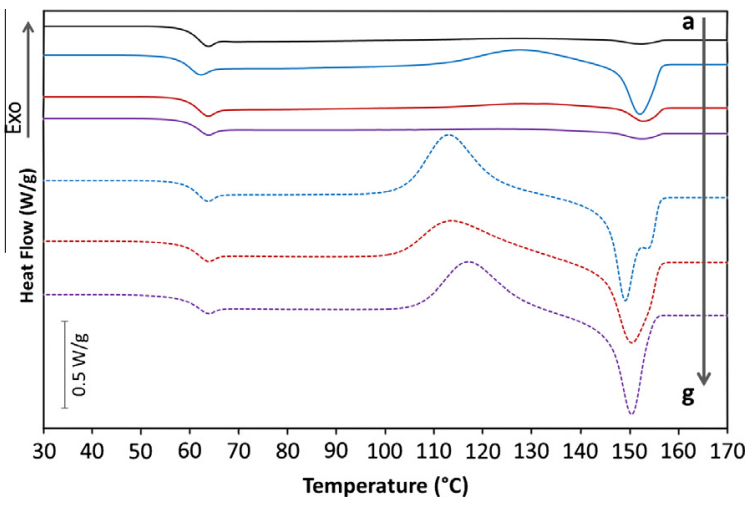

Fig. 5. Second heating run thermograms for (a) neat PLA and PLA reinforced with CNW at (b) $2.5 \mathrm{wt} \%$, (c) $7.5 \mathrm{wt} \%$ and (d) $15 \mathrm{wt} \%$, and CNWICN at (e) $2.5 \mathrm{wt} \%$, (f) $7.5 \mathrm{wt} \%$ and (g) $15 \mathrm{wt} \%$.

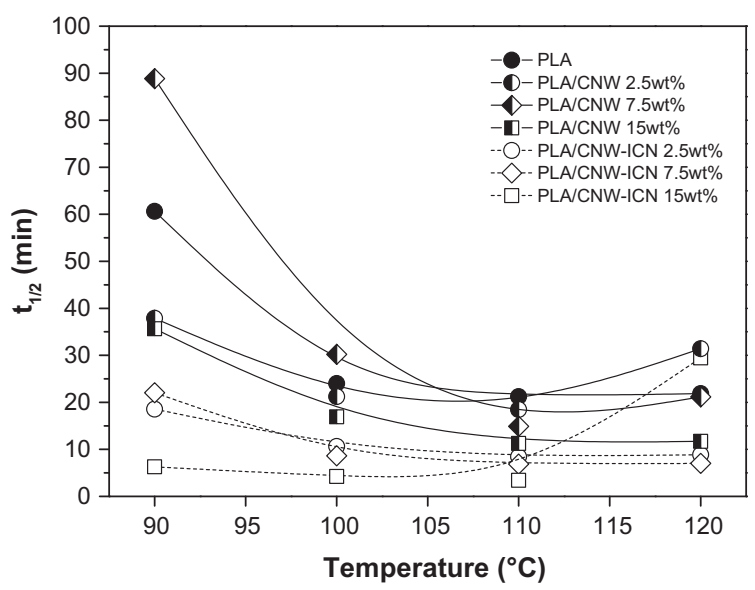

Fig. 6. Comparison of the crystallization half times dertermined by DSC for different annealing conditions for PLA and bionanocomposite films. Lines are drawn to guide the eye.

published data $[46,52,53]$. The action of a nucleating agent is the reduction of the nucleation induction period and the increase of the number of primary nucleation sites. Fig. 6 shows that unmodified CNW had a small (15 wt\%) or no effect (2.5 and $7.5 \mathrm{wt} \%$ ) on the crystallization rate of PLA. This result has also been observed by Pei et al. [42]. As expected from data reported in Fig. 5, CNW-ICN had a clear evidence of nucleating effect, which increased with its concentration. The biggest difference was observed at $100^{\circ} \mathrm{C}$. The $t_{1 / 2}$ of PLA was reduced from 25 min to 8.4, 6.9 and $3.4 \mathrm{~min}$ using $2.5,7.5$ and $15 \mathrm{wt} \%$ of CNW-ICN, respectively. A similar effect has been recently published for PCL/CNW composite [10]. The nucleating efficiency of CNW-ICN was equal to the efficiency of talc, which is a very widely used nucleating material for PLA $[45,46,51,53-55]$ and to the efficiency of silylated CNW [42]. Inorganic nanofillers showed higher acceleration of the PLA crystallization kinetics. For example Day et al. obtained a decrease in $t_{1 / 2}$ from 21 min to only one minute with the help of a specially designed nanocomposite [50]. An interesting result was obtained by Krikorian and
Pochan [56]. They compared the nucleating efficiency of different organomodified nanoclays and showed that the most compatible nanoclay had the smallest effect. A certain incompatibility can thus favour nucleation. This is not in complete accordance with what happened in our case with nanocellulose. This might be due to the fact that the number of interface/contact filler with the matrix is much more important once chemical modified $\mathrm{CNW}$ are used contrary to $\mathrm{CNW}$ and its aggregates. One might issue that CNW was completely incompatible and that the surface grafting brought about just the slight increase of compatibility needed for inducing the nucleating effect.

The equilibrium melting temperatures of pure PLA and PLA bionanocomposites were obtained using the HoffmanWeeks method, the corresponding experimental data are listed in Table 2. Data linearization and extrapolation were used to determinate the stability parameters, $\varphi, T_{m}^{0}$ based on the least square method, and the thickening coefficient, $\gamma$, which represent the ratio of the lamellar thickness to the lamellar thickness of the critical nucleus [57]. $T_{m}^{0}$ for PLA amounted to $175^{\circ} \mathrm{C}$, which is consistent with Courgneau et al. $\left(173.5^{\circ} \mathrm{C}\right)$ [53]. Differences are linked to different percentages of D-lactic acid in PLA [58]. In the bionanocomposites, the equilibrium melting point was not decreased and stability parameters were not affected when unmodified CNW is used like reinforcement. This result showed the non-compatibility of CNW and PLA.

For grafted CNW, losing of correlation and no melting point depression is observed. Therefore, no prediction is possible about the influence of the nanoparticles inside the polymer following the Hoffman-Weeks method. Furthermore after isothermal crystallization the glass transition temperature of CNW-ICN decreased in comparison with the Tg before crystallization, while it remained constant in the case of CNW. CNW-ICN might thermally degrade during the isothermal crystallization (carried out during $3 \mathrm{~h}$ ) and the degradation products can have caused plasticizing. The effect was more important for higher CNW-ICN concentrations and higher crystallization temperature. Melting point depression of the CNW-ICN bionanocomposites could therefore stem from two different causes and the Hoffman-Weeks model could not be applied anymore.

\subsection{Mechanical and thermomechanical properties}

Tensile properties are a very sensitive probe for interfacial adhesion in a composite, as in general strength and

Table 2

Equilibrium melting temperature $\left(T_{m}^{0}\right)$, stability parameter $(\varphi)$ and thickening coefficient $(\gamma)$ obtained from Hoffman-Weeks plots of PLA and PLA bionanocomposites.

\begin{tabular}{llrrl}
\hline Sample & $T_{m}^{0}\left({ }^{\circ} \mathrm{C}\right)$ & \multicolumn{1}{l}{$\varphi$} & \multicolumn{1}{l}{$\gamma$} & $R^{2}$ \\
\hline PLA & 175 & 0.38 & 2.6 & 0.97 \\
PLA/CNW 2.5 wt\% & 173 & 0.34 & 3.0 & 0.99 \\
PLA/CNW 7.5 wt\% & 175 & 0.39 & 2.6 & 0.99 \\
PLA/CNW 15 wt\% & 175 & 0.38 & 2.6 & 0.99 \\
PLA/CNW-ICN 2.5 wt\% & 172 & 0.35 & 2.8 & 0.91 \\
PLA/CNW-ICN 7.5 wt\% & 172 & 0.36 & 2.8 & 0.84 \\
PLA/CNW-ICN 15 wt\% & 140 & -0.07 & -14.8 & 0.35 \\
\hline
\end{tabular}


elongation at break decrease when interfacial affinity is not ensured [42,59-61]. Films of PLA and its bionanocomposites were evaluated by tensile testing in order to investigate the effect of CNW and CNW-ICN on mechanical properties of the composites. Mechanical properties as tensile strength and elongation at break are shown in Table 3. The mechanical tests of neat PLA showed its very brittle behaviour and are within the range of values published in different literature studies, which are between 30 and $60 \mathrm{MPa}[26,42,53,59,62-64]$. Statistical analysis showed that the addition of non-compatible CNW imparted mechanical properties, in that both tensile strength and elongation at break decreased. Sanchez-Garcia and Lagaron [15] showed the same lack of interaction between CNW and PLA inducing a reduction of mechanical properties. A reduction in tensile strength between $36 \%$ and 56\% was also reported. On the same way Petersson and Oksman [40] reported a high decrease in mechanical properties (16\% for elongation at break) when they used microcrystalline cellulose ( $5 \mathrm{wt} \%$ ) as reinforcement in PLA. On the contrary, the mechanical properties were improved when $\mathrm{CNW}$-ICN was incorporated. Improvement was higher at lower concentrations; the best result was achieved by an increase of roughly $10 \mathrm{MPa}$ for $2.5 \mathrm{wt} \% \mathrm{CNW}-\mathrm{ICN}$. Further increase of CNW-ICN quantity did not yield improvement of tensile strength according to neat PLA, where else the highest elongation at break was obtained at the higher CNW-ICN concentration. Habibi and Dufresne [65] obtained similar results when adding neat and surface modified CNW into PCL. Siqueira et al. [34] also observed similar positive effect of chemically grafted CNW with PCL. This reinforcement with grafted CNW is classically explained by a better dispersion and a better compatibility with the matrix, but always a compromise with the CNW network quality has to be found.

Indeed, the mechanism of mechanical reinforcement of CNW is also to rely on hydrogen bonding between nanoparticles at concentrations above the percolation threshold [2]. The percolation threshold in our case lies at approximately $2.7 \mathrm{wt} \%$ [3]. In some cases a decrease of mechanical properties can also be observed when the CNW surface is grafted as the grafts disturb the hydrogen bonding [66]. In our case, however, due to the incompatibility between filler and matrix this effect is less important than the compatibilisation and dispersion enhancement thanks to the grafting. Moreover as shown by contact angle and FTIR, the grafting is not very efficient and still some $\mathrm{OH}$ groups are available for hydrogen bonded network.

The storage modulus E' provides information on thermo-mechanical behaviour and interactions due to the incorporation of CNW or CNW-ICN within the PLA matrix. Fig. 7 shows the temperature dependence of $E^{\prime}$ of the neat PLA matrix and related nanocomposites. As it is well known, the exact determination of the glassy modulus depends on the precise knowledge of the sample dimensions which is subjected to high uncertainty in the case of very thin film samples as ours. In order to minimize this influence and to underline differences in behaviour, the curves were arbitrarily shifted to coincide at the glassy elastic modulus of PLA at $0{ }^{\circ} \mathrm{C}$. To separate the reinforcing effect of the PLA degree of crystallinity from the effect of the nanowhiskers, the neat PLA and bionanocomposites were crystallized inside the DMA equipment at $110^{\circ} \mathrm{C}$ during 20 min before recording $E^{\prime}$. The neat recrystallized PLA displayed a typical behaviour of semicrystalline polymer with three different zones: the glassy plateau $\left(T<52{ }^{\circ} \mathrm{C}\right)$, the glass transition $\left(52<T<80^{\circ} \mathrm{C}\right)$, and the rubbery plateau before melting and flow starting at $130^{\circ} \mathrm{C}$. For comparison reasons, the $E^{\prime}$ curve of an amorphous PLA which was not recrystallized in the DMA equipment is also plotted in Fig. 7a. It shows that the PLA modulus in the rubbery plateau rises to the same value than the modulus of the recrystallized PLA. Nanocomposites reinforced with CNW at 2.5 and $15 \mathrm{wt} \%$ did not change the film behaviour compared to neat PLA, but probably for different reasons. Indeed the concentration of $2.5 \mathrm{wt} \%$ is below percolation threshold, so the hydrogen bonding mechanism cannot occur, and at $15 \mathrm{wt} \%$ the very big aggregates and poor interfacial adhesion did not allow for reinforcement either. In the case of CNW at 7.5 wt\%, an action of the percolated CNW network could be possible. On the contrary, the compatibilized CNW-ICN nanofiller obtained an increase in the rubbery modulus, increase which was higher at higher concentration. The crystallization enthalpy of the different samples annealed at $110^{\circ} \mathrm{C}$ from the melt is given in Table 1 . It remained the same for all CNW-ICN containing samples.

Table 3

Dynamic mechanical analysis, oxygen permeability and water vapour transmission rate coefficients for PLA and PLA bionanocomposites.

\begin{tabular}{|c|c|c|c|c|}
\hline \multirow[t]{2}{*}{ Sample } & \multicolumn{2}{|c|}{ Mechanical properties } & \multicolumn{2}{|l|}{ Barrier properties } \\
\hline & $\begin{array}{l}\text { Tensile strength } \\
(\mathrm{MPa})\end{array}$ & $\begin{array}{l}\text { Elongation at break } \\
(\%)\end{array}$ & $\begin{array}{l}\mathrm{O}_{2} \\
\text { permeability }\left(\times 10^{18} \mathrm{~m}^{3} \mathrm{~m} \mathrm{~m}^{-2} \mathrm{~s}^{-1} \mathrm{~Pa}^{-1}\right)\end{array}$ & $\begin{array}{l}\mathrm{H}_{2} \mathrm{O} \text { permeability } \\
\left(\times 10^{14} \mathrm{~kg} \mathrm{~m} \mathrm{~s}^{-1} \mathrm{~m}^{-2} \mathrm{~Pa}\right)\end{array}$ \\
\hline PLA & $40 \pm 1^{\mathrm{a}}$ & $4.3 \pm 0.3^{\mathrm{b}, \mathrm{c}}$ & $2.9 \pm 0.6^{\mathrm{a}}$ & $2.200 \pm 0.045^{\mathrm{a}}$ \\
\hline PLA/CNW 2.5\% & $40 \pm 1^{\mathrm{a}}$ & $5.3 \pm 0.6^{\mathrm{b}}$ & $3.3 \pm 1.4^{\mathrm{a}}$ & $2.671 \pm 0.222^{\mathrm{b}}$ \\
\hline PLA/CNW 7.5\% & $32 \pm 2^{b}$ & $4.0 \pm 0.8^{c}$ & $2.9 \pm 0.4^{\mathrm{a}}$ & $3.134 \pm 0.410^{c}$ \\
\hline PLA/CNW 15\% & $15 \pm 1^{\mathrm{c}}$ & $3.4 \pm 0.4^{\mathrm{c}}$ & $4.3 \pm 0.7^{\mathrm{a}}$ & $6.507 \pm 0.42^{\mathrm{d}}$ \\
\hline $\begin{array}{l}\text { PLA/CNW-ICN } \\
2.5 \%\end{array}$ & $51 \pm 2^{\mathrm{d}}$ & $5.6 \pm 1.2^{\mathrm{b}}$ & $3.2 \pm 1.0^{\mathrm{a}}$ & $2.367 \pm 0.035^{\mathrm{a}, \mathrm{b}}$ \\
\hline $\begin{array}{l}\text { PLA/CNW-ICN } \\
\quad 7.5 \%\end{array}$ & $44 \pm 1^{\mathrm{e}}$ & $5.4 \pm 0.3^{b}$ & $3.9 \pm 0.3^{a}$ & $2.428 \pm 0.062^{\mathrm{a}, \mathrm{b}}$ \\
\hline $\begin{array}{l}\text { PLA/CNW-ICN } \\
\quad 15 \%\end{array}$ & $47 \pm 2^{f}$ & $7.7 \pm 0.9^{\mathrm{a}}$ & $3.3 \pm 0.8^{\mathrm{a}}$ & $2.298 \pm 0.008^{\mathrm{a}, \mathrm{b}}$ \\
\hline
\end{tabular}

\footnotetext{
a,b,c,d Significant differences at $p<0.05$ (Duncan).
} 


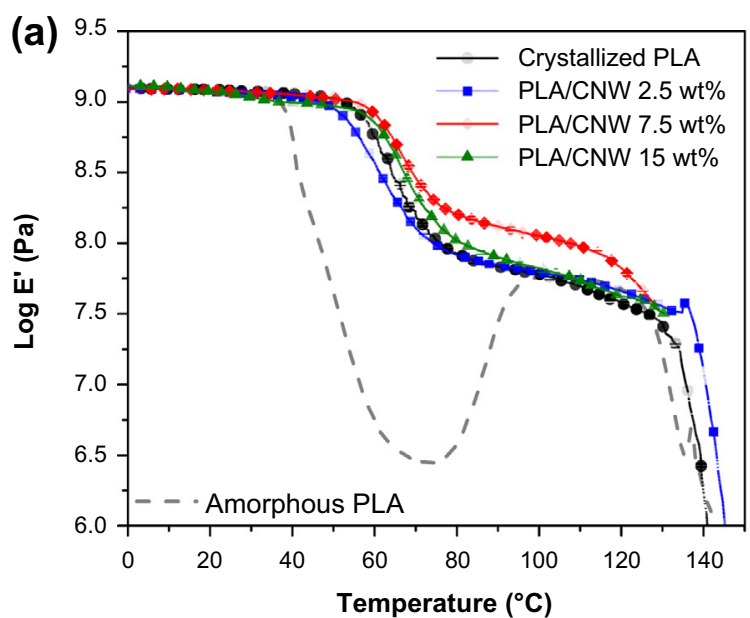

(b)

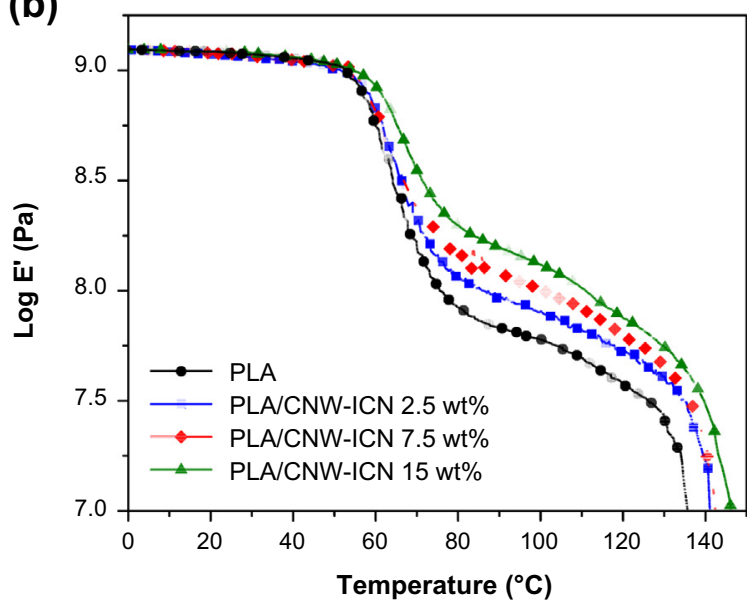

Fig. 7. Logarithm of the storage tensile modulus $\left(E^{\prime}\right)$ for neat PLA and bionanocomposites reinforced with (a) CNW and (b) CNW-ICN at 2.5, 7.5 and $15 \mathrm{wt} \%$.

Furthermore, the crystallization enthalpy of the CNW-ICN nanocomposites cold crystallization, also displayed in Table 1, showed no differences. We suppose therefore, that the crystallinity degree of the samples after heat treatment in the DMA equipment showed no high differences. Therefore, we think that the increase in $E$ ' of the bionanocomposites could be attributed to the nanowhiskers presence.

\subsection{Barrier properties of bionanocomposites films}

Table 3 gathers the oxygen and water vapour permeabilities of PLA as function of the nanofiller content. The oxygen permeability values of the blank samples are comparable to literature values laying around $2.4 \times 10^{-18} \mathrm{~m}^{3}$ $\mathrm{m} \mathrm{m}^{-2} \mathrm{~s}^{-1}[53,62,67-70]$, although films were prepared by casting. The water permeability is also in the range of literature data $[15,62,71]$. High variance of the results of the oxygen permeability was observed, most likely linked to the heterogeneous character of the samples. Statistical analysis of $\mathrm{O}_{2}$ permeability showed therefore no significant difference between the neat polymer and PLA/CNW and
PLA/CNW-ICN nanocomposites. The PLA/CNW 15 wt\% had maybe slightly higher oxygen permeability, most probably due to poor interfacial adhesion and aggregate presence. These results indicate that barrier properties to oxygen of the PLA were not reduced but not bettered either by the addition of the nanofiller. Petersson and Oksman [40] reported an increase of $241 \%$ in oxygen permeability attributed to the lack of good interaction of the microcrystalline cellulose with the PLA. On the contrary, SanchezGarcia and Lagaron [15] reported a substantial decrease in $\mathrm{O}_{2}$ permeability (reduction by $90 \%$ ) upon introduction of CNW. However, the comparison of results shows that the blank value $\left(1.37 \times 10^{-17} \mathrm{~m}^{3} \mathrm{~m} \mathrm{~m}^{-2} \mathrm{~s}^{-1} \mathrm{~Pa}^{-1}\right)$ of Sanchez-Garcia and Lagaron [15] was one order of magnitude higher than ours and their results for the nanocomposites are in the same range than ours (between 1.6 and $\left.2.3 \times 10^{-18} \mathrm{~m}^{3} \mathrm{~m} \mathrm{~m}^{-2} \mathrm{~s}^{-1} \mathrm{~Pa}^{-1}\right)$. The water vapour permeability values of the PLA bionanocomposites were higher when CNW were used compared with CNW-ICN, and the water vapour permeability increased according to the CNW content $(21.3 \%, 42.4 \%$ and $195.8 \%)$. No significant difference was found in for the CNW-ICN samples. We assume that inclusion of very hydrophilic structures in PLA increased the water solubility coefficient. These results are in agreement with Sanchez-Garcia et al. [11] where an increase of $44.3 \%$ and $80.0 \%$ of water vapour transfer was observed when PLA was reinforced with 5 and $10 \mathrm{wt} \%$ of cellulose fibres. It can be concluded that the grafting of CNW successfully counteracted this effect. Test at temperatures higher than glass transition could be interesting to investigate the performance in the rubbery polymer, but was not possible with our equipment.

\section{Conclusion}

A novel, in situ grafting method was applied in the aim of producing surface modified CNW with enhanced compatibility with PLA. Successful grafting of CNW by $n$-octadecyl isocyanate was evidenced and CNW-ICN could be satisfyingly dispersed in the PLA matrix. The grafting induced compatibility, which provided CNW-ICN with a nucleating effect on PLA comparable to talc. CNW-ICN showed however some thermal degradation under severe conditions, which for its thermal stability needs therefore to be investigated further. The enhanced compatibility brought about increased tensile strength of the bionanocomposites at low CNW-ICN concentrations and higher thermal resistance of PLA, as shown by an increased rubbery plateau modulus. Oxygen permeability was not affected by the blending with CNW-ICN. More interestingly, the water vapour permeability of CNW-ICN bionanocomposities was lower than the one of the comparable CNW bionanocomposites. This led to the conclusion that hydrophobic grafting might counteract the expected effect of enhanced water vapour permeability due to inclusion of hydrophilic structures in the matrix. In conclusion, the surface grafting of CNW with a long chain aliphatic molecule was successful in providing compatibilized structures and could be, with regards to the variety of different iscocyanates available, a easy method for designing functional, 
fully organic and eventually biodegradable bionanocomposites based on PLA.

\section{References}

[1] Habibi Y, Lucia LA, Rojas OJ. Cellulose nanocrystals: chemistry, selfassembly, and applications. Chem Rev 2010;110(6):3479-500.

[2] Favier V, Chanzy H, Cavaille JY. Polymer nanocomposites reinforced by cellulose whiskers. Macromolecules 1995;28(18):6365-7.

[3] Siqueira G, Bras J, Dufresne A. Cellulosic bionanocomposites: a review of preparation, properties and applications. Polymers 2010;2(4):728-65.

[4] Visakh PM, Thomas S, Oksman K, Mathew AP. Cellulose nanofibres and cellulose nanowhiskers based natural rubber composites: diffusion, sorption, and permeation of aromatic organic solvents. J Appl Polym Sci 2012;124(2):1614-23.

[5] Siqueira G, Abdillahi H, Bras J, Dufresne A. High reinforcing capability cellulose nanocrystals extracted from Syngonanthus nitens (Capim Dourado). Cellulose 2010;17(2):289-98.

[6] Mathew AP, Dufresne A. Morphological investigation of nanocomposites from sorbitol plasticized starch and tunicin whiskers. Biomacromolecules 2002;3(3):609-17.

[7] Grunert M, Winter W. Nanocomposites of cellulose acetate butyrate reinforced with cellulose nanocrystals. J Polym Environ 2002;10(12):27-30.

[8] Azizi Samir MAS, Alloin F, Sanchez JY, Dufresne A. Cellulose nanocrystals reinforced poly(oxyethylene). Polymer 2004;45(12): 4149-57.

[9] Jalal Uddin A, Araki J, Gotoh Y. Toward "strong" green nanocomposites: polyvinyl alcohol reinforced with extremely oriented cellulose whiskers. Biomacromolecules 2011;12(3): 617-24.

[10] Siqueira G, Fraschini C, Bras J, Dufresne A, Prud'Homme R, Laborie M-P. Impact of the nature and shape of cellulosic nanoparticles on the isothermal crystallization kinetics of poly(epsilon-caprolactone). Eur Polym J 2011:47(12):2216-27.

[11] Sanchez-Garcia MD, Gimenez E, Lagaron JM. Morphology and barrier properties of solvent cast composites of thermoplastic biopolymers and purified cellulose fibers. Carbohydr Polym 2008;71(2):235-44.

[12] Ten E, Jiang L, Wolcott MP. Preparation and properties of aligned poly(3-hydroxybutyrate-co-3-hydroxyvalerate)/cellulose nanowhiskers composites. Carbohydr Polym 2013;92(1):206-13.

[13] Ten E, Jiang L, Wolcott MP. Crystallization kinetics of poly(3hydroxybutyrate-co-3-hydroxyvalerate)/cellulose nanowhiskers composites. Carbohydr Polym 2013;90(1):541-50.

[14] Mathew AP, Oksman K, Sain M. The effect of morphology and chemical characteristics of cellulose reinforcements on the crystallinity of polylactic acid. J Appl Polym Sci 2006;101(1):300-10.

[15] Sanchez-Garcia M, Lagaron J. On the use of plant cellulose nanowhiskers to enhance the barrier properties of polylactic acid. Cellulose 2010;17(5):987-1004.

[16] Goffin A-L, Raquez J-M, Duquesne E, Siqueira G, Habibi Y, Dufresne A, et al. From interfacial ring-opening polymerization to melt processing of cellulose nanowhisker-filled polylactide-based nanocomposites. Biomacromolecules 2011;12(7):2456-65.

[17] Liu X, Khor S, Petinakis E, Yu L, Simon G, Dean K, et al. Effects of hydrophilic fillers on the thermal degradation of poly(lactic acid). Thermochim Acta 2010;509(1-2):147-51.

[18] Madhavan Nampoothiri K, Nair NR, John RP. An overview of the recent developments in polylactide (PLA) research. Bioresource Technol 2010;101(22):8493-501.

[19] Domenek S, Courgneau C, Ducruet V. Characteristics and applications of poly(lactide). John Wiley \& Sons Inc.; 2011. p. 183223.

[20] Auras RA, Loong-Tak Lim, Susan EM, Tsuji H. Poly(lactic acid): synthesis, structures, properties, processing, and applications. New York: John Wiley \& Sons; 2011.

[21] Avérous L. In: Belgacem MN, Gandini A, editors. Polylactic acid: synthesis, properties and applications. Elsevier Limited, Publication; 2008. p. 433-50.

[22] Shi Q, Zhou C, Yue Y, Guo W, Wu Y, Wu Q. Mechanical properties and in vitro degradation of electrospun bio-nanocomposite mats from PLA and cellulose nanocrystals. Carbohydr Polym 2012;90(1):301-8.

[23] Siro I, Plackett D. Microfibrillated cellulose and new nanocomposite materials: a review. Cellulose 2010;17(3):459-94.

[24] Suryanegara L, Nakagaito A, Yano H. Thermo-mechanical properties of microfibrillated cellulose-reinforced partially crystallized PLA composites. Cellulose 2010;17(4):771-8.
[25] Graupner N, Herrmann AS, Müssig J. Natural and man-made cellulose fibre-reinforced poly(lactic acid) (PLA) composites: an overview about mechanical characteristics and application areas. Compos A Appl Sci Manuf 2009;40(6-7):810-21.

[26] Oksman K, Mathew AP, Bondeson D, Kvien I. Manufacturing process of cellulose whiskers/polylactic acid nanocomposites. Compos Sci Technol 2006;66(15):2776-84.

[27] Kowalczyk M, Piorkowska E, Kulpinski P, Pracella M. Mechanical and thermal properties of PLA composites with cellulose nanofibers and standard size fibers. Compos A Appl Sci Manuf 2011;42(10): 1509-14.

[28] Frone AN, Berlioz S, Chailan JF, Panaitescu DM, Donescu D. Cellulose fiber-reinforced polylactic acid. Polym Compos 2011;32(6): 976-85.

[29] Beck S, Bouchard J, Berry R. Dispersibility in water of dried nanocrystalline cellulose. Biomacromolecules 2012;13(5):1486-94.

[30] Çetin NS, Tingaut P, Özmen N, Henry N, Harper D, Dadmun M, et al. Acetylation of cellulose nanowhiskers with vinyl acetate under moderate conditions. Macromol Biosci 2009;9(10):997-1003.

[31] Lin N, Huang J, Chang PR, Feng J, Yu J. Surface acetylation of cellulose nanocrystal and its reinforcing function in poly(lactic acid). Carbohydr Polym 2011;83(4):1834-42.

[32] Braun B, Dorgan J, Knauss D. Reactively compatibilized cellulosic polylactide microcomposites. J Polym Environ 2006;14(1):49-58.

[33] Braun B, Dorgan JR, Hollingsworth LO. Supra-molecular ecobionanocomposites based on polylactide and cellulosic nanowhiskers: synthesis and properties. Biomacromolecules 2012; 13(7):2013-9.

[34] Siqueira G, Bras J, Dufresne A. New process of chemical grafting of cellulose nanoparticles with a long chain isocyanate. Langmuir 2010;26(1):402-11.

[35] Belgacem MN, Gandini A. Chemical modification of wood in monomers, polymers and composites from renewable resources. Elsevier; 2008.

[36] Bondeson D, Mathew A, Oksman K. Optimization of the isolation of nanocrystals from microcrystalline cellulose by acid hydrolysis. Cellulose 2006;13(2):171-80.

[37] Fischer EW, Sterzel HJ, Wegner G. Investigation of the structure of solution grown crystals of lactide copolymers by means of chemical reactions. Colloid Polym Sci 1973;251(11):980-90.

[38] Ramires EC, Dufresne A. A review of cellulose nanocrystals and nanocomposites. Tappi J 2011:9-16.

[39] Jebrane M, Sèbe G, Cullis I, Evans PD. Photostabilisation of wood using aromatic vinyl esters. Polym Degrad Stab 2009;94(2):151-7.

[40] Petersson L, Oksman K. Biopolymer based nanocomposites: comparing layered silicates and microcrystalline cellulose as nanoreinforcement. Compos Sci Technol 2006;66(13):2187-96.

[41] Fortunati E, Armentano I, Zhou Q, Puglia D, Terenzi A, Berglund LA, et al. Microstructure and nonisothermal cold crystallization of PLA composites based on silver nanoparticles and nanocrystalline cellulose. Polym Degrad Stab 2012;97(10):2027-36.

[42] Pei A, Zhou Q, Berglund LA. Functionalized cellulose nanocrystals as biobased nucleation agents in poly(l-lactide) (PLLA) - crystallization and mechanical property effects. Compos Sci Technol 2010;70(5): 815-21.

[43] Fortunati E, Armentano I, Iannoni A, Barbale M, Zaccheo S, Scavone $\mathrm{M}$, et al. New multifunctional poly(lactide acid) composites: mechanical, antibacterial, and degradation properties. J Appl Polym Sci 2012;124(1):87-98.

[44] Fortunati E, Armentano I, Zhou Q, Iannoni A, Saino E, Visai L, et al Multifunctional bionanocomposite films of poly(lactic acid), cellulose nanocrystals and silver nanoparticles. Carbohydr Polym 2012;87(2):1596-605.

[45] Di Lorenzo ML. Crystallization behavior of poly(L-lactic acid). Eur Polymer J 2005;41(3):569-75.

[46] Li H, Huneault MA. Effect of nucleation and plasticization on the crystallization of poly(lactic acid). Polymer 2007;48(23):6855-66.

[47] De Santis F, Pantani R, Titomanlio G. Nucleation and crystallization kinetics of poly(lactic acid). Thermochim Acta 2011;522:128-34.

[48] Courgneau C, Ducruet V, Avérous L, Grenet J, Domenek S. Nonisothermal crystallization kinetics of poly(lactide)-effect of plasticizers and nucleating agent. Polym Eng Sci 2013;53(5): 1085-98.

[49] Picard E, Espuche E, Fulchiron R. Effect of an organo-modified montmorillonite on PLA crystallization and gas barrier properties. Appl Clay Sci 2011;53(1):58-65.

[50] Day M, Nawaby A, Liao X. A DSC study of the crystallization behaviour of polylactic acid and its nanocomposites. J Therm Anal Calorim 2006;86(3):623-9. 
[51] Di Lorenzo ML. The crystallization and melting processes of poly(Llactic acid). Macromol Symp 2006;234(1):176-83.

[52] Li Y, Chen C, Li J, Sun XS. Isothermal crystallization and melting behaviors of bionanocomposites from poly(lactic acid) and $\mathrm{TiO}_{2}$ nanowires. J Appl Polym Sci 2012;124(4):2968-77.

[53] Courgneau C, Domenek S, Lebossé R, Guinault A, Avérous L, Ducruet $V$. Effect of crystallization on barrier properties of formulated polylactide. Polym Int 2012;61(2):180-9.

[54] Li M, Hu D, Wang Y, Shen C. Nonisothermal crystallization kinetics of poly(lactic acid) formulations comprising talc with poly(ethylene glycol). Polym Eng Sci 2010;50(12):2298-305.

[55] Xiao H, Liu F, Jiang T, Yeh J-T. Kinetics and crystal structure of isothermal crystallization of poly(lactic acid) plasticized with triphenyl phosphate. J Appl Polym Sci 2010;117(5):2980-92.

[56] Krikorian V, Pochan DJ. Unusual crystallization behavior of organoclay reinforced poly(L-lactic acid) nanocomposites. Macromolecules 2004;37(17):6480-91.

[57] Hoffman JD, Weeks JJ. Melting process and the equilibrium melting temperature of polychlorotrifluoroethylene. J Res Natl Bureau Stand 1962;66A:13-28.

[58] Saeidlou S, Huneault MA, Li H, Park CB. Poly(lactic acid) crystallization. Prog Polym Sci 2012;37(12):1657-77.

[59] Mathew AP, Oksman K, Sain M. Mechanical properties of biodegradable composites from poly lactic acid (PLA) and microcrystalline cellulose (MCC). J Appl Polym Sci 2005;97(5): 2014-25.

[60] Iwatake A, Nogi M, Yano H. Cellulose nanofiber-reinforced polylactic acid. Compos Sci Technol 2008;68(9):2103-6.

[61] Bondeson D, Oksman K. Dispersion and characteristics of surfactant modified cellulose whiskers nanocomposites. Compos Interfaces 2007;14(7-9):617-30.

[62] Courgneau C, Domenek S, Guinault A, Avérous L, Ducruet V. Analysis of the structure-properties relationships of different multiphase systems based on plasticized poly(lactic acid). J Polym Environ 2011;19(2):362-71.

[63] Coltelli M-B, Maggiore ID, Bertoldo M, Signori F, Bronco S, Ciardelli F. Poly(lactic acid) properties as a consequence of poly(butylene adipate-co-terephthalate) blending and acetyl tributyl citrate plasticization. J Appl Polym Sci 2008;110(2):1250-62.

[64] Elangovan D, Yuzay IE, Selke SEM, Auras R. Poly(L-lactic acid) metal organic framework composites: optical, thermal and mechanical properties. Polym Int 2012;61(1):30-7.

[65] Habibi Y, Dufresne A. Highly filled bionanocomposites from functionalized polysaccharide nanocrystals. Biomacromolecules 2008;9(7):1974-80.

[66] Dufresne A. Processing of polymer nanocomposites reinforced with polysaccharide nanocrystals. Molecules 2010;15(6):4111-28.

[67] Colomines G, Ducruet V, Courgneau C, Guinault A, Domenek S. Barrier properties of poly(lactic acid) and its morphological changes induced by aroma compound sorption. Polym Int 2010;59(6): $818-26$.

[68] Guinault A, Sollogoub C, Ducruet V, Domenek S. Impact of crystallinity of poly(lactide) on helium and oxygen barrier properties. Eur Polym J 2012;48(4):779-88.

[69] Sawada H, Takahashi Y, Miyata SKS, Sato S, Nagai K. Gas transport properties and crystalline structure of poly(lactic acid) membranes. Trans Mater Res Soc Jpn 2010;35:241-6.

[70] Drieskens M, Peeters R, Mullens J, Franco D, Lemstra PJ, HristovaBogaerds DG. Structure versus properties relationship of poly(lactic acid). I. Effect of crystallinity on barrier properties. J Polym Sci, Part B: Polym Phys 2009;47(22):2247-58.

[71] Delpouve N, Stoclet G, Saiter A, Dargent E, Marais S. Water barrier properties in biaxially drawn poly(lactic acid) films. J Phys Chem B 2012;116(15):4615-25. 